\title{
A Review of Studies on Ethnic Differences of Brain-Derived Neurotrophic Factor Genes in Patients with Post-Traumatic Stress Disorder
}

\author{
Liumei Luo', Juncheng Guo'², Xiang Li ${ }^{3}$, Min Guo', Ping Huang4 \\ ${ }^{1}$ Hainan General Hospital, Sanya, China \\ ${ }^{2}$ Haikou Hospital Affiliated to Xiangya School of Medicine, Central South University, Haikou, China \\ ${ }^{3}$ The Third People's Hospital of Hubei Province, Wuhan, China \\ ${ }^{4}$ University of South China, Hengyang, China \\ Email: ^g2002m@163.com
}

How to cite this paper: Luo, L.M., Guo, J.C., Li, X., Guo, M. and Huang, P. (2019) A Review of Studies on Ethnic Differences of Brain-Derived Neurotrophic Factor Genes in Patients with Post-Traumatic Stress Disorder. World Journal of Neuroscience, 9, 229-242.

https://doi.org/10.4236/wjns.2019.94017

Received: August 14, 2019

Accepted: September 24, 2019

Published: September 27, 2019

Copyright (c) 2019 by author(s) and Scientific Research Publishing Inc. This work is licensed under the Creative Commons Attribution International License (CC BY 4.0).

http://creativecommons.org/licenses/by/4.0/

\begin{abstract}
In the regulation of human nervous system, cognitive function and other genes, epigenetics changes the expression of genes after being influenced by the external environment. DNA methylation levels are also different in different ethnic groups, and a large number of studies have shown that post-traumatic stress disorder (PTSD) has a certain genetic predisposition. Through the national differences of PTSD brain-derived neurotrophic factor genes, it not only provides new research directions for the pathogenesis and treatment of 5-HT-related mental diseases, but also provides information and new genetic indicators for forensic personal identification, paternity testing and assessment of mental status. A review studies on the national differences of brain-derived neurotrophic factor genes in patients with post-traumatic stress disorder.
\end{abstract}

\section{Keywords}

Ethnicity, Post-Traumatic Stress Disorder, Brain-Derived Neurotrophic Factor, Gene Methylation

\section{Introduction}

The characteristics of the distribution of various ethnic groups in China are: large scattered, small settlements, and mixed living. There are ethnic minorities living in the Han area, and Han people living in ethnic minority areas [1]. This pattern of distribution is formed by the interaction and flow of various ethnic groups in the long-term historical development process. This pattern of distri- 
bution is formed by the interaction and flow of various ethnic groups in the long-term historical development process. Although China's ethnic minority population is small, it is widely distributed [2]. All provinces, autonomous regions, and municipalities directly under the Central Government have ethnic minorities, and most county-level units have more than two ethnic groups. China's ethnic minorities are mainly distributed in Inner Mongolia, Xinjiang, Ningxia, Guangxi, Tibet, Yunnan, Guizhou, Qinghai, Sichuan, Gansu, Liaoning, Jilin, Hunan, Hubei, Hainan, Taiwan and other provinces and autonomous regions [3]. All provinces, autonomous regions, and municipalities directly under the Central Government have ethnic minorities, and most county-level units have more than two ethnic groups. China's ethnic minorities are mainly distributed in Inner Mongolia, Xinjiang, Ningxia, Guangxi, Tibet, Yunnan, Guizhou, Qinghai, Sichuan, Gansu, Liaoning, Jilin, Hunan, Hubei, Hainan, Taiwan and other provinces and autonomous regions [4]. Hainan is a multi-ethnic province. In addition to the Han nationality, there are 53 ethnic minorities. Among the 53 ethnic minorities, the Li ethnic group has the largest population, followed by the Miao, Zhuang and Hui nationalities. The total population of Hainan Province is $8,334,400$, of which the Han population is $6,885,500$, accounting for $82.6 \%$ of the total population; the minority population is $1,448,900$, accounting for $17.4 \%$ of the total population (including the Li population of 1,319,100, the Miao, 68,400, and Hui people 10.7 million people) [4].

The Han and Li nationalities are the two major ethnic groups in Hainan. The Li nationality is a unique ethnic minority in Hainan and a representative ethnic minority group in China. Hainan Island is surrounded by the sea and belongs to the tropical ocean monsoon climate. Its unique climate, geographical conditions and historical relationship have left the Li people still retaining the unique customs of ethnic minorities. At the same time, Hainan Island is separated from the mainland of the motherland in terms of its geographical environment, with less population migration and a special genetic background. Due to genetic factors, ethnic differences, regional environment, etc., there may be differences in genetic genes of different races; different populations may have different responsiveness to these virulence factors. Affected by many factors, the Li ethnic group in Hainan may differ from other regions or other ethnic groups in the incidence, pathogenesis and prognosis of certain diseases. DNA methylation is one of the important ways to express epigenetic mechanisms. In the regulation of human nervous system and cognitive function, epigenetics changes the expression of genes after being influenced by the external environment. DNA methylation levels are also different in different ethnic groups, and a large number of studies have shown that post-traumatic stress disorder (PTSD) has a certain genetic predisposition [5] [6] [7]. Recently, some scholars have proposed a multi-gene inheritance method in which multiple micro-effect genes work synergistically. It is also suggested that PTSD is the result of the independent action of a single main effector gene or other genetic and environmental factors. At present, there 
are few reports on the methylation of PTSD and brain-derived neurotrophic factor (BDNF) gene. For this reason, we use case-control based on the epidemiological investigation of PTSD in the Li and Han nationalities in Hainan Province. Correlation analysis method, using polymerase chain reaction (PCR) and pyrophosphate sequencing method to detect DNA methylation of CpG island of BDNF gene, comparing Li and Han patients diagnosed as PTSD in Hainan Province and normal $\mathrm{Li}$ and Han populations Detection of the level of methylation of the BDNF gene was performed. There are few reports on the methylation of PTSD and brain-derived neurotrophic factor (BDNF) gene. To this end, based on the PTSD epidemiological survey of the $\mathrm{Li}$ and Han nationalities in Hainan Province, we used the case-control correlation analysis method to detect the DNA methylation of the CPG island of BDNF gene by polymerase chain reaction (PCR) and pyrophosphate sequencing. We tested the methylation level of BDNF gene in Li and Han patients diagnosed as PTSD in Hainan Province and normal $\mathrm{Li}$ and Han control populations. To analyze the relationship between the methylation level of BDNF gene and the pathogenesis of PTSD, and whether the methylation level of BDNF gene is different between $\mathrm{Li}$ and Han in Hainan Province, and explore the susceptibility factors of PTSD in Li nationality. These studies can help to have a deeper understanding of the pathogenesis of PTSD, provide basic information for the pathogenesis of PTSD and its prevention and treatment, and provide genetic information for anthropological, forensic identification and PTSD association studies.

\section{Post-Traumatic Stress Disorder (PTSD)}

In recent years, with the increase of natural disasters, wars, terrorist incidents, etc., research on post-traumatic stress disorder (PTSD) has been increasing. The magnitude 8.0 earthquake that occurred in Wenchuan, Sichuan Province, China on May 12, 2008 has further promoted the attention and research on PTSD. Post-traumatic stress disorder is a delayed and long-lasting mental disorder caused by unusual, threatening or catastrophic trauma [8]. Its specific symptoms include re-experience, avoidance, and increased alertness following traumatic events. The diagnostic criteria must include a specific number of each of the three types of symptoms: re-experience, avoidance, and increased vigilance [9]. In addition, the definition of the source of PTSD stress has made the necessary major changes based on existing research results. It was confined to an unusual war incident at first, and gradually expanded to withstand major disasters (large earthquakes, shipwrecks, floods, big bangs, mine disasters, fires, terrorist incidents, traffic accidents, etc.), physical attacks, violent sexual assaults, etc. In recent years, clinical psychology has also regarded life-threatening diseases as one of the stressors that cause PTSD, such as cancer. Many medical events such as surgery, maternal production, abortion, etc. can also lead to PTSD. The concept of stressors now extends to special occupations, emotional crises, criminal offences, etc. [10]. Epidemiological survey results show that wars, terrorist attacks, 
major natural disasters such as earthquakes, major mine disasters, etc. can lead to PTSD (3.7\% to 60\%) [11]. Foreign data showed that for men, the incidence of high incidence of PTSD in human trauma was rape (65\%), war (38.8\%), sudden death of the lover (12.6\%), and harassment (12.2\%); For women, rape (45.9\%), harassment (26.5\%), physical assault (21.3\%), and sudden death of the lover (16.2\%) [1]. According to statistics, the incidence of PTSD after 3 months of car accidents was $38.27 \%$. It can be seen that in addition to the severe stress events of the population, individuals suffering from severe stress events can also trigger PTSD, and with the frequent occurrence of various traumatic events, the incidence of PTSD is on the rise. Therefore, PTSD research has received more and more attention. Due to special geographical factors, natural disasters such as typhoons, floods, earthquakes, tsunamis and fires occur frequently in Hainan Province. As a national border defense and military base area, it is also threatened by a series of disasters such as chemical poisoning, explosions, epidemics, major sea, land and air traffic accidents, and terrorist activities. Unconventional emergencies can lead to PTSD, and PTSD research has become a hot issue worldwide. Long-term PTSD can cause depression, anxiety, suicidal tendencies, schizophrenia and many other problems, and it is often accompanied by problems such as unemployment, family instability, natural disasters, and health emergencies, which have serious social harm. Among many PTSD genetic susceptibility candidate genes, the research group pays close attention to brain-derived neurotrophic factor (BDNF) gene, serotonin transporter gene promoter polymorphism (5-HTTLPR), and whether there is a difference between the two levels in the Li and Han PTSD patients in Hainan Province. The paper is explored for research progress (review), with no study grouping and correlation.

\section{Brain-Derived Neurotrophic Factor (BDNF)}

Animal experiments and neurobiochemistry, neuroendocrine and other studies have suggested that BDNF may be related to the occurrence of PTSD, and may also participate in the occurrence of PTSD. First, existing studies have shown that BDNF is involved in the pathogenesis of a variety of mental disorders, and PTSD has a certain degree of commonality with regard to pathogenesis, clinical manifestations and genetic susceptibility [12]. Second, BDNF is expected to become a new target for prognosis and gene therapy for a variety of mental illnesses and brain trauma [13]. DNF is the most abundant neurotrophic factor in the body. Its expression is mainly located in the cerebral cortex, hippocampus, striatum, etc. It plays an important role in brain development, can affect the axial growth and connection of neurons, and mediate the proliferation of neurons, differentiation and survival [14]. Overexpression in the central nervous system plays an important role in promoting anxiety [15]. In the central nervous system, BDNF is mainly synthesized in neurons, transported by antegrade axoplasmic to axon terminals, and released by high-affinity receptor tyrosine protein kinase B (TrkB) to target cells. In addition, BDNF can also be secreted by target 
cells that act on neurons, and reverse trophic neurons. Immunohistochemistry confirmed that in the central nervous system, BDNF immunopositive neurons were widely distributed in the rat brain, especially in the hippocampus, thalamus striatum and cortex. In the hippocampus, BDNF mRNA content is 20 - 30 times higher than NGF mRNA. In the peripheral nervous system, more BDNF appeared in the distal part of the nerve ending after nerve injury. In the ovary, heart, lung, and skeletal muscles other than nerve tissue, BDNF is also expressed in a small amount. BDNF is highly abundant in human plasma, while BDNF in whole blood is localized in platelets, but the source of BDNF in platelets is unclear. Due to the high content of BDNF in the blood, studies on the repair and regeneration of peripheral nervous system by BDNF have been focused. Some areas with weak blood-brain barriers such as the hypothalamus may have BDNF through the blood-brain barrier, so the mutual sensibility of peripheral blood and nerve center BDNF is not excluded. It is necessary to explore whether the change of BDNF content in peripheral blood can be used as a direct indicator of anti-psychiatric diseases.

Mayfair measured the microRNA content of brain cells treated with BDNF and compared it with microRNA content in neurons that were not stimulated with BDNF [16]. The researchers found that the level of certain microRNAs decreased after administration of BDNF to brain cells. This result suggests that BDNF regulates the levels of these microRNAs, which in turn affects protein production. BDNF has a wide and important role in the growth, development, differentiation, regeneration and functional maintenance of various types of neurons, and is essential for regulating hippocampal synaptic plasticity and memory. Clinically, it is found that the fear memory of patients with PTSD is difficult to subside or the fear memory has gradually reappeared, suggesting that fear memory regression can be the key to the occurrence and treatment of PTSD. The research on the mechanism of fear memory regression needs to be further studied. The hippocampus is an important brain region for learning and memory. The long-term potentiation effect (LTP) of neurons in the hippocampus is the neural basis of long-term memory. Studies have shown that blocking the expression of BDNF in hippocampus during long-term memory formation has caused long-term memory consolidation defects, but does not affect the formation of long-term memory, indicating that the normal expression of BDNF has a memory consolidation and long-term retention. Very critical role. Injection of recombinant human BDNF into the hippocampus reverses the long-term memory deficit caused by the protein synthesis inhibitor anisomycin, and BDNF antisense nucleotides are blocked in the hippocampus of rats with conditional fear to block BDNF expression. Later, its fear response to conditional cues weakened, indicating that BDNF is a key factor affecting the long-term maintenance of fear memory [17] [18]. R Vulturar et al. reported the distribution of the BDNF Val66Met allele in a sample of healthy volunteers $(\mathrm{N}=1124)$ selected from Romanians. The frequency of the Val allele was $80.74 \%$ and the frequency 
of the Met allele was 19.26\% [19]. The data expansion efforts of this study mapped the allele distribution of BDNF Val66Met populations around the world, and emphasized population stratification applied in future studies, reporting phenotypic association control in samples of different populations. CHY $\mathrm{Fu}, \mathrm{MacGregor} \mathrm{Legge} \mathrm{et} \mathrm{al.} \mathrm{focused} \mathrm{on} \mathrm{the} \mathrm{contribution} \mathrm{of} \mathrm{the} \mathrm{brain-derived}$ neurotrophic factor (BDNF) Val66Met polymorphism to major depressive disorder and attempted to determine whether the same neurological effects were observed in healthy individuals [20]. There is a specific focus on the amygdala, the anterior cingulate cortex in the prefrontal cortex and the cortical thickness of the middle frontal and temporal frontal cortex. The study focused on 79 patients diagnosed with major depressive disorder and 74 control participants. The main effect was the thinning of the cortex of the mid-frontal cortex in the tail of patients with major depressive disorder and the control group. The polymorphism in the frontal cortex was greater in the caudate cortex, and no significant interaction was found in the amygdala. In Caucasians, the Val allele is found to be the most common, and the frequency of the Met allele is 25\% to 32\%. In Asian populations, the Met allele is more frequent, about $40 \%$ to $50 \%$. Since MacGregor Legge et al. found that the Met allele has the greatest impact on major depressive disorder, we believe that it is crucial to examine how different frequencies affect the occurrence of the disorder. Ivac et al. found that polymorphisms and mood disorders may depend on race in their racial differences in BDNF Val66Met polymorphisms in non-clinical samples from Croatia and Korea. Studies that searched for BDNF polymorphisms in Carribbean and other races in Africa did not yield results. Further research on this topic will be important as generalized conclusions about the differential effects of BDNF polymorphisms on major depressive disorder or any major psychiatric disorder of different ethnic groups cannot be derived. T Andreska et al. performed a polymorphism study on BDNF gene Val66Met (rs6265) in elderly Han depression patients, and found that their genotypes were significantly different between patients and controls, and the frequency of Met alleles was significantly higher than that of the control group. It is believed that this polymorphism may be involved in the occurrence of depression and is a risk factor for elderly patients with depression in China [21]. Peters et al. divided the rats with conditional fear regression training into the regressive success group and the regression failure group according to the level of regression. The BDNF concentration difference between the two groups was compared by ELISA, and it was found that the regression was compared with the regression failure group. The level of BDNF in the hippocampus of the rats in the successful group was significantly increased [22]. Although animal experiments and neurobiochemical studies suggest that BDNF may be involved in the development of PTSD, no BDNF gene mutation has been found that is directly related to the pathogenesis of PTSD. A study of 106 patients with Korean PTSD and 161 healthy controls did not find a association between the BDNF gene Val66Met polymorphism and PTSD [23]. Zhang et al. 
found that three SNPs of BDNF gene (G-712A, C270T, Val66Met) were associated with susceptibility to PTSD in a small sample of PTSD patients [24]. In other studies of mental illness, animal experiments found that BDNF gene protein levels and mRNA levels were reduced in depressive model rats, while long-term electroconvulsive therapy increased BDNF gene protein expression in hippocampus and frontal cortex [25]. Autopsy studies found that the expression of BDNF in the hippocampus of patients with PTSD was significantly lower than that of the normal control group, while the expression of BDNF in the dentate gyrus of patients who had received antidepressant treatment was significantly higher than that of the untreated patients [26]. It has been reported that the serum BDNF level of patients with severe PTSD is significantly lower than that of the normal control group. Treatment with antidepressant or electroconvulsive without convulsion can significantly increase serum BDNF levels, and the associated PTSD is significantly reduced. BDNF may mediate mood disorders, including post-traumatic stress disorder. Huang et al. performed a polymorphism study on BDNF gene Val66Met (rs6265) in elderly Han PTSD patients, and found that the genotype was significantly different between patients and the control group, and the frequency of Met allele was significantly higher than that of the control group. Polymorphism may be involved in the occurrence of PTSD and is a risk factor for elderly PTSD patients in China [27]. Strauss et al reported that the $\mathrm{T}$ allele of BDNF gene rs7103411 is significantly associated with childhood onset PTSD [28]. There are many associations between BDNF gene polymorphism and schizophrenia. Spalletta and other studies have found that BDNF Val66Met polymorphism is associated with schizophrenia symptoms, especially impulsive behavior [29]. Zintzaras et al.'s study of 877 patients with schizophrenia and 989 controls showed that the BDNF gene C270T polymorphism T carriers had a $63 \%$ increased risk of schizophrenia compared with homozygous $\mathrm{C} / \mathrm{C}$ carriers $(\mathrm{OR}=11.63)$, support for the association of this polymorphism with schizophrenia [30]. Studies by H Ji et al. have shown that BDNF C270T is a risk factor for Alzheimer's disease in Asia, and BDNF is involved in the pathogenesis of various psychiatric diseases [31]. At present, it is preliminarily speculated that BDNF is a candidate gene for psychiatric diseases such as PTSD and schizophrenia, but there are reports of inconsistent correlation results. The frequency of distribution of BDNF gene $(\mathrm{C} 270 \mathrm{~T}) \mathrm{C} / \mathrm{T}, \mathrm{T} / \mathrm{T}$ genotype and $\mathrm{T}$ allele frequency in children with schizophrenia were significantly higher than those in the control group. One of the main reasons for the inconsistency of the above findings may be the heterogeneity of schizophrenia [32]. Studies by Yuichiro Watanabe and others have shown associations between the $\mathrm{T}$ allele and schizophrenia [33].

Therefore, the correlation between BDNF gene polymorphism and PTSD requires a more in-depth analysis of expression levels, and requires a larger sample size and other regional and ethnic basic data to be confirmed in different ethnic groups, regions and populations. 


\section{BDNF Gene Expression of PTSD}

Y Liu et al. discussed the relationship between 5-HTTLPR polymorphism and the diagnosis of combat exposure and post-traumatic stress disorder (PTSD). The results indicate a link between 5-HTTLPR genotype and PTSD diagnosis in NHB veterans [34]. NA Kimbrel et al. discussed the 186-returned Iraqi and Afghan veterans genotyped as a 5-HTTLPR polymorphism, and as a result, veterans carrying the 5-HTTLPR polymorphism of the $S$ allele may face an increased risk of adjustment problems, and Reduce the quality of life after deployment to the theater [35]. The results of RH Pietrzak et al. showed that the low expression variant of the 5-HTTLPR polymorphism modifies the risk of PTSD, but this effect may be specific anxiety arousal and re-experience symptoms [36]. MJ Telch et al. measured 5-HTTLPR, a biomarker that is susceptible to stress in war zones, which is essential for developing more effective treatment and prevention strategies for soldiers and civilians exposed to trauma [37]. These data suggest that the 5-HTTLPR genotype can modulate individual sensitivity to war zone stressors and expression of mood disorders, including PTSD symptoms. This association of replication, as well as the identification of other risk genetic regulators, can drive the development of biomarkers that can predict relative elasticity and vulnerability to stress. Haixia Long's findings suggest that there are differences between Caucasian and Han subjects in the association between different forms of 5-HTTLPR and DMN (brain default state network) functional connections [38]. Noskova et al. found that racial differences in the allele frequency of 5-HTTLPR exist between Caucasians and Asians [39]. The study investigated racial differences in 5-HTTLPR of 1804 healthy Caucasian populations from several European countries in Croatia and the Russian Federation. Due to the low frequency of S alleles and S/S genotypes, the genotype and allele frequencies of 5-HTTLPR are significantly different between male and female Croatians, Russians, monks and Bashkir $(\mathrm{P}<0.001)$. Russians have slightly different allele and genotype distributions, and Bashkir and Tatar have similar allele and genotype frequencies. Compared with Russians, Croats have a higher frequency of S/S genotypes among deaf and Bashkir people. Gender-related differences occur only in the allelic distribution in the Bashkir population. These racial differences may lead to inconsistent results in association studies between different mental disorders, personality traits, behaviors, and 5-HTTLPR between different races, and should be controlled to enable the results to be disseminated among different populations. Brett C studied 5HTTLPR and rs25531 polymorphisms in six samples from North America, Southeast Asia, and Africa (Cameroon). These samples differed in race and ethnic composition, identifying allele and genotype frequencies for 24,066 participants. The results showed that the frequency of the rs $25531 \mathrm{G}$ allele was higher in black and African populations than in Caucasian, Hispanic and Asian populations [40]. In addition, we observed a larger number of ultra-long ("XL") 5-HTTLPR alleles than previously reported, compared to the total lack of Caucasian and Native American popula- 
tions, Asian, black and non-white Spanish. Almost alleles are almost completely present in the population. In the final joint consideration, we observed sample differences in genotype frequencies in ethnic and racial populations. Taken together, these data highlight the importance of characterizing L-G alleles to avoid misclassification of participants by genotypes and to further investigate the possible impact of XL alleles on the transcriptional efficiency of SLC6A4.

\section{National Differences in Brain-Derived Neurotrophic Factor Genes in Patients with Post-Traumatic Stress Disorder}

The gene determines the gene product--the primary sequence of the protein, and then the other chemical structures in the primary sequence form other high-level structures to form a specific function of the protein, and the gene regulatory protein is essential for regulating gene replication and transcription. Almost everyone is beginning to realize that both genetics and the environment play a role in individual development, and that extreme emphasis on the role of one factor cannot be more fully explained. The current research has set aside the controversy between innate and acquired, and has begun to focus on how genetics interact with the environment. This role transcends the interpretation of any single factor, and the methodological meaning of a holistic view and dialectical connection is reflected. Developmental scholars Scoel and McCartney propose three ways of interacting with the environment, namely, passive genotype-environment-related, evoked genotype-environment-related and active genotype-environmental relationships. LR Dougherty et al. explored whether the interaction between brain-derived neurotrophic factor (BDNF) Val66Met polymorphism is associated with stress response of the hypothalamic-pituitary adrenal (HPA) axis. Community samples of 144 preschool children were genotyped and exposed to stress-inducing laboratory tasks. Salivary cortisol was obtained at four time points before and after the standardized laboratory assessment, involving pressure sources separated from parents and frustration tasks. Children homozygous for the 5-HTTLPR allele carrying the Met-BDNF allele demonstrated a significant decrease in the initial level of cortisol, followed by a positive increase in cortisol in response to laboratory stressors. In contrast, children homozygous for the 5-HTTLPR and Val-BDNF alleles demonstrated a greater decrease in cortisol in response to laboratory stressors. The results suggest a link between the regulation of 5-HTTLPR by BDNF gene and the biological stress response in children. The epistatic effect plays a role in individual differences in stress regulation and may be associated with genetic vulnerability of stress-related diseases [41]. Peter C and colleagues studied whether there was a relationship between polymorphisms in the serotonin transporter (SLC6A4, 5-HTTLPR) and brain-derived neurotrophic factor (BDNF Val66Met, rs6265) genes and moderate life stress and rumination. The results showed that individuals with two 5-HTTLPR polymorphisms of the short (S) allele or two Met al- 
leles of the BDNF Val66Met polymorphism under life stress conditions were more abundant than other genotypes. In addition, the accumulation of risk alleles (i.e. S and Met alleles) across genes is associated with greater ruminance in the context of life stress [42]. These results indicate a relationship between 5 HTTLPR and BDNF Val66Met and life stress and ruminant. These findings support the notion that the variation of these genes is associated with the biological sensitivity to the negative effects of stress [43] [44]. At present, the research on the pathogenesis of PTSD is still in the experimental stage. The research on BDNF believes that it is widely involved in the pathogenesis of PTSD, and plays an important role in various hypotheses of PTSD, such as the hypotrophic hypothesis of neurotrophic factors and monoamine hypothesis. Studies have shown that BDNF is a molecular marker of serotonin neurons in the central nervous system, which promotes the regeneration of neurons, and the two molecules interact at the molecular level. Therefore, the relevance of BDNF and PTSD needs to be further studied.

\section{Outlook and Conclusion}

Genetic imprinting is a category of epigenetics (igenetics) research, unlike genetic studies of general significance. Genetic research in a general sense usually involves changes in the function of genes caused by changes in the sequence of DNA such as mutations, deletions, and the like. The study of epigenetics focuses on the absence of DNA sequence changes, DNA or chromosomes which have changed their original function through modification. Recently, some scholars have suggested that PTSD is the result of the independent action of a single main effector gene or other genetic and environmental factors. There are few reports on the methylation of PTSD and brain-derived neurotrophic factor (BDNF) gene, especially among ethnic groups. To this end, based on the PTSD epidemiological survey of the Li and Han nationalities in Hainan Province, we examined the methylation level of BDNF gene in patients with PTSD diagnosed by Li and Han in Hainan Province and normal Li and Han to analyze the relationship between the methylation level of BDNF gene and the pathogenesis of PTSD, and whether there are differences among the ethnic groups in $\mathrm{Li}$ and Han in Hainan Province, explore the susceptibility factors of PTSD in Li nationality in Hainan Province, and provide a basis for the research and prevention of PTSD pathogenesis. The data provide a scientific basis for anthropological, forensic identification and PTSD related research.

\section{Conflicts of Interest}

The authors declare no conflicts of interest regarding the publication of this paper.

\section{References}

[1] Tam, G., Chan, E.Y.Y. and Liu, S. (2019) Planning of a Health Emergency Disaster 
Risk Management Programme for a Chinese Ethnic Minority Community. International Journal of Environmental Research and Public Health, 16, E1046. https://doi.org/10.3390/ijerph16061046

[2] Zhang, F., Zheng, H.C. and Liu, C.J. (2019) Forensic Features for Yunnan Lisu Ethnic Minority and Phylogenetic Structure Exploration among 26 Chinese Populations. International Journal of Legal Medicine, 133, 103-104. https://doi.org/10.1007/s00414-018-1930-5

[3] Liu, J., Wang, Z., He, G.L., Wang, M.G. and Hou, Y.P. (2019) Genetic Polymorphism and Phylogenetic Differentiation of the Huaxia Platinum System in Three Chinese Minority Ethnicities. Scientific Reports, 9, Article No. 3371.

https://doi.org/10.1038/s41598-019-39794-y

[4] Ding, Y.P., Niu, H., Yang, H., Sun, P., Chen, Y., Duan, M.L., Xu, D.C., Xu, J.X. and Jin, T.B. (2015) EGLN2 and RNF150 Genetic Variants Are Associated with Chronic Obstructive Pulmonary Disease Risk in the Chinese Population. International Journal of Chronic Obstructive Pulmonary Disease, 10, 145-151. https://doi.org/10.2147/COPD.S73031

[5] Schner, J., Heinz, A., Endres, M., Gertz, K. and Kronenberg, G. (2017) Post-Traumatic Stress Disorder and Beyond: An Overview of Rodent Stress Models. Journal of Cellular and Molecular Medicine, 21, 2248-2256. https://doi.org/10.1111/jcmm.13161

[6] Mehta, D. and Binder, E.B. (2012) Gene $\times$ Environment Vulnerability Factors for PTSD: The HPA-Axis. Neuropharmacology, 62, 654-662. https://doi.org/10.1016/j.neuropharm.2011.03.009

[7] Lambert, K.G., Nelson, R.J., Jovanovic, T. and Cerdá, M. (2015) Brains in the City: Neurobiological Effects of Urbanization. Neuroscience and Biobehavioral Reviews, 58, 107-122. https://doi.org/10.1016/j.neubiorev.2015.04.007

[8] Lin, X.B., Qi, J.R., Li, Z.Q., et al. (2014) Analysis of 347 Pairs of Couples with Intermediate or Severe Thalassemia Genetic Risk in Hainan Rural Area. Hainan Medicine, 22, 3397-3401.

[9] Svenaeus, F., et al. (2013) Diagnosing Mental Disorders and Saving the Normal. American Psychiatric Association, 11, 197-120.

[10] McCarron, K.K., Reinhard, M.J., Bloeser, K.J., Mahan, C.M. and Kang, H.K. (2014) PTSD Diagnoses among Iraq and Afghanistan Veterans: Comparison of Administrative Data to Chart Review. Journal of Traumatic Stress, 5, 626-629.

https://doi.org/10.1002/jts.21961

[11] Schwarzer, R., Cone, J.E., Li, J., Bowler, R.M., et al. (2016) A PTSD Symptoms Trajectory Mediates between Exposure Levels and Emotional Support in Police Responders to 9/11: A Growth Curve Analysis. BMC Psychiatry, 16, 201. https://doi.org/10.1186/s12888-016-0907-5

[12] Li, L.J. and Yu, X. (2013) Introduction to the Guidelines for Prevention and Treatment of Posttraumatic Stress Disorder. Chinese Journal of Neurology, 46, 196-198.

[13] Joy, D.M., et al. (2013) Genomic and Epigenomic Insights into Nutrition and Brain Disorders. Nutrients, 5, 887-914. https://doi.org/10.3390/nu5030887

[14] Notaras, M., Hill, R., Van, B.M., et al. (2015) The BDNF Gene Val66Met Polymorphism as a Modifier of Psychiatric Disorder Susceptibility: Progress and Controversy. Molecular Psychiatry, 20, 916-930. https://doi.org/10.1038/mp.2015.27

[15] Han, E.J., Kim, Y.K., Hwang, J., Kim, S.H., Lee, H.J., et al. (2015) Evidence for Association between the Brain-Derived Neurotrophic Factor Gene and Panic Disorder: A Novel Haplotype Analysis. Psychiatry Investigation, 12, 112-117. 
https://doi.org/10.4306/pi.2015.12.1.112

[16] Sakharnova, T.A., Vedunova, M.V., Mukhina, I.V., et al. (2012) Brain-Derived Neurotrophic Factor (BDNF) and Its Role in the Functioning of the Central Nervous System. Neurochemical Journal, 6, 251-259. https://doi.org/10.1134/S1819712412030129

[17] BioVal.Bioon.com (2017) Issue of Cell Magazine: 2017 Brain Science and Brain Intelligence Symposium.

[18] Lee, B., Sur, B., Yeom, M., et al. (2016) Effects of Systemic Administration of Ibuprofen on Stress Response in a Rat Model of Post-Traumatic Stress Disorder. Korean Journal of Physiology \& Pharmacology, 20, 357-366. https://doi.org/10.4196/kjpp.2016.20.4.357

[19] Zhang, L., Li, X.X., Hu, X.Z., et al. (2016) Post-Traumatic Stress Disorder Risk and Brain-Derived Neurotrophic Factor Val66Met. World Journal of Psychiatry, 6, 553-557. https://doi.org/10.5498/wjp.v6.i1.1

[20] Vulturar, R., Chiş, A., Hambrich, M., Kelemen, B., Ungureanu, L., et al. (2016) Allelic Distribution of BDNF Val66Met Polymorphism in Healthy Romanian Volunteers. Translational Neuroscience, 7, 31-34. https://doi.org/10.1515/tnsci-2016-0006

[21] Fu, C.H.Y., Legge, M.G., et al. (2015) Ethnic Differences in BDNF Val66Met Polymorphism Reply. British Journal of Psychiatry, 207, 363-364. https://doi.org/10.1192/bjp.207.4.363a

[22] Andreska, T., Aufmkolk, S., Sauer, M., Blum, R., et al. (2014) High Abundance of BDNF within Glutamatergic Presynapses of Cultured Hippocampal Neurons. Frontiers in Cellular Neuroscience, 8, 1411-1443. https://doi.org/10.3389/fncel.2014.00107

[23] Peters, J., Dieppa-Perea, L.M., Melendez, L.M., et al. (2010) Induction of Fear Extinction with Hippocampal-Infralimbic BDNF. Science, 328, 1288-1290. https://doi.org/10.1126/science.1186909

[24] Lee, H.Y., Kim, Y.K., et al. (2008) Plasma Brain-Derived Neurotrophic Factor as Peripheral Marker for the Action Mechanism of Antidepressant. Neuropsychobiology, 57, 194-199. https://doi.org/10.1159/000149817

[25] Zhang, H., Ozbay, F., Lappalainen, J., et al. (2009) Brain Derived Neurotrophic Factor (BDNF) Gene Variants and Alzheimer's Disease, Affective Disorders, Posttraumatic Stress Disorder, Schizophrenia, and Substance Dependence. American Journal of Medical Genetics. Part B, Neuropsychiatric Genetics, 141, 387-393. https://doi.org/10.1002/ajmg.b.30332

[26] Dong, Y.X., Sun, X.H., Yue, H.E., et al. (2014) The Expression of Hippocampal BDNF, TrkB and p75NTR in Depression Model Rats and the Effects of Mirtazapine. Journal of Brain \& Nervous Diseases, 3, 302-306.

[27] Chen, B., Dowlatshahi, D., MacQueen, G.M., et al. (2010) Increased Hippocampal BDNF Immunoreactivity in Subjects Treated with Antidepressant Medication. Biological Psychiatry, 50, 260-265. https://doi.org/10.1016/S0006-3223(01)01083-6

[28] Hwang, J.P., Tsai, S.J., Hong, C.J., et al. (2006) The Val66Met Polymorphism of the Brain-Derived Neurotrophic Factor Gene Is Associated with Geriatric Depression. Neurobiology of Aging, 27, 1834-1837. https://doi.org/10.1016/j.neurobiolaging.2005.10.013

[29] Strauss, J., Barr, C.L., George, C.J., et al. (2008) Brain-Derived Neurotrophic Factor (BDNF) Variants Are Associated with Childhood-on-Set Mood Disorder: Confirmation in a Hungarian Sample. Molecular Psychiatry, 10, 861-867. 
https://doi.org/10.1038/sj.mp.4001685

[30] Spalletta, G., Morris, D.W., Angelucci, F., et al. (2010) BDNF Val66Met Polymorphism Is Associated with Aggressive Behavior in Schizophrenia. European Psychiatry, 25, 311-313. https://doi.org/10.1016/j.eurpsy.2009.10.008

[31] Zintzaras, E., et al. (2007) Brain-Derived Neurotrophic Factor Gene Polymorphisms and Schizophrenia: A Meta Analysis. Psychiatric Genetics, 17, 69-75. https://doi.org/10.1097/YPG.0b013e32801119da

[32] Ji, H., Dai, D., Wang, Y., Jiang, D., Zhou, X., et al. (2015) Association of BDNF and BCHE with Alzheimer's Disease: Meta-Analysis Based on 56 Genetic Case-Control Studies of 12, 563 Cases and 12, 622 Controls. Experimental \& Therapeutic Medicine, 9, 1831-1840. https://doi.org/10.3892/etm.2015.2327

[33] Martinotti, G., Sepede, G., Brunetti, M., Ricci, V., Gambi, F., et al. (2015) BDNF Concentration and Impulsiveness Level in Post Traumatic Stress Disorder. Psychiatry Research, 229, 814-818. https://doi.org/10.1016/j.psychres.2015.07.085

[34] Watanabe, Y., Nunokawa, A., et al. (2013) Association of the BDNF C270T Polymorphism with Schizophrenia: Updated Meta-Analysis. Psychiatry \& Clinical Neurosciences, 67, 123-125. https://doi.org/10.1111/pcn.12018

[35] Kim, E.-J. and Kim, Y.-K. (2018) 196G/A of the Brain-Derived Neurotrophic Factor Gene Polymorphisms Predicts Suicidal Behavior in Schizophrenia Patients. Psychiatry Investigation, 15, 733-738. https://doi.org/10.30773/pi.2018.02.27

[36] Liu, Y. and Garrett, M.E. (2015) An Examination of the Association between 5-HTTLPR, Combat Exposure, and PTSD Diagnosis among U.S. Veterans. PLoS ONE, 10, e0119998. https://doi.org/10.1371/journal.pone.0119998

[37] Kimbrel, N.A., Morissette, S.B., Meyer, E.C., Chrestman, R., Jamroz, R., et al. (2014) Effect of the 5-HTTLPR Polymorphism on Posttraumatic Stress Disorder, Depression, Anxiety, and Quality of Life among Iraq and Afghanistan Veterans. Anxiety Stress \& Coping, 28, 456-466. https://doi.org/10.1080/10615806.2014.973862

[38] Pietrzak, R.H., Galea, S., Southwick, S.M., Gelernter, J., et al. (2013) Examining the Relation between the Serotonin Transporter 5-HTTPLR Genotype X Trauma Exposure Interaction on a Contemporary Phenotypic Model of Posttraumatic. Journal of Affective Disorders, 148, 123-128. https://doi.org/10.1016/j.jad.2012.11.003

[39] Telch, M.J., Beevers, C.G., David, R., Han-Joo, L., Albert, R., et al. (2015) 5-HTTLPR Genotype Potentiates the Effects of War Zone Stressors on the Emergence of PTSD, Depressive and Anxiety Symptoms in Soldiers Deployed to Iraq. World Psychiatry Official Journal of the World, 14, 198-206. https://doi.org/10.1002/wps.20215

[40] Long, H.X., Liu, B., et al. (2014) A Potential Ethnic Difference in the Association between 5-HTTLPR Polymorphisms and the Brain Default Mode Network. Chinese Science Bulletin, 13, 1355-1361. https://doi.org/10.1007/s11434-014-0185-x

[41] Pivac, N.T., Nedić, N., Kazantseva, G., Gaysina, A., et al. (2008) Ethnic Differences in the Serotonin Transporter Polymorphism (5-HTTLPR) in Several European Populations. Progress in Neuro-Psychopharmacology \& Biologic, 32, 1735-1739. https://doi.org/10.1016/j.pnpbp.2008.07.012

[42] Haberstick, B.C., Smolen, A., et al. (2015) Population Frequencies of the Triallelic 5-HTTLPR in Six Ethnically Diverse Samples from North America, Southeast Asia, and Africa. Behavior Genetics, 45, 255-261. https://doi.org/10.1007/s10519-014-9703-5

[43] Dougherty, L.R., Klein, D.N., Congdon, E., Canli, T., Hayden, E.P., et al. (2010) Interaction between 5-HTTLPR and BDNF Val66Met Polymorphisms on HPA Axis 
Reactivity in Preschoolers. Biological Psychology, 83, 93-100.

https://doi.org/10.1016/j.biopsycho.2009.10.009

[44] Clasen, P.C., Wells, T.T., et al. (2011) 5-HTTLPR and BDNF Val66Met Polymorphisms Moderate Effects of Stress on Rumination. Genes, Brain and Behavior, 10, 740-746. https://doi.org/10.1111/j.1601-183X.2011.00715.x 